Applications

\title{
ForestScanner: A mobile application for measuring and mapping trees with LiDAR- equipped iPhone and iPad
}

Shinichi Tatsumi ${ }^{1, *}$, Keiji Yamaguchi ${ }^{2}$, and Naoyuki Furuya ${ }^{1}$

${ }^{1}$ Hokkaido Research Center, Forestry and Forest Products Research Institute, Hokkaido, Japan

${ }^{2}$ Mapry Inc., Hyogo, Japan

*Corresponding author: Shinichi Tatsumi

Address: Hokkaido Research Center, Forestry and Forest Products Research Institute, Hitsujigaoka 7, Toyohira-ku, Sapporo, Hokkaido 062-8516, Japan

Email: $\quad$ community.ecologist@gmail.com

ORCID: $\quad 0000-0002-1789-1685$

Running headline: Terrestrial laser scanning with iPhone/iPad 


\section{ABSTRACT}

2 1. Terrestrial laser scanning (TLS) is becoming increasingly popular as an alternative means to

3 conventional forest inventory methods. By gauging the distances to multiple points on the

$4 \quad$ surrounding object surfaces, TLS acquires 3D point clouds from which tree sizes and spatial

5 distributions can be rapidly estimated. However, the high cost and specialized skills required

6 for TLS have put it out of reach for many potential users.

7 2. We here introduce ForestScanner, a free, mobile application that allows TLS-based forest inventories by means of iPhone or iPad with a built-in LiDAR sensor. ForestScanner does not require any manual analysis of 3D point clouds. As the user scans trees with an iPhone/iPad, ForestScanner estimates the stem diameters and spatial coordinates based on real-time object detection and circle fitting. The users can visualize, check, and share the

12 results of scanning in situ.

13 3. By using ForestScanner, we measured the stem diameters and spatial coordinates of 672 trees within a 1 ha plot in 1 h 39 min with an iPhone and in 1 h 38 min with an iPad (diameter $\geq 5$ $\mathrm{cm}$; detection rate $=100 \%$ ). ForestScanner reduced the person-hours required for measuring diameters to $25.7 \%$, mapping trees to $9.3 \%$, and doing both to $6.8 \%$ of the person-hours taken using a dimeter tape and a conventional surveying method. The diameters measured by

ForestScanner and diameter tape were in good agreement; $\mathrm{R}^{2}=0.963$ for iPhone and ha subplots); Mantel $\mathrm{R}^{2}=0.999$ for both iPhone and iPad.

4. Our results indicate that ForestScanner enables cost-, labor-, and time-efficient forest inventories. The application can increase the accessibility to TLS for people beyond 
24 specialists and enhance resource assessments and biodiversity monitoring in forests

25 worldwide.

26

27 Keywords: Augmented reality, Diameter at breast height, Forest inventory, Light detection and

28 ranging, Mobile phone, Remote sensing, Smartphone, Terrestrial laser scanning 


\section{1 | INTRODUCTION}

30 Forest inventory has been an essential component of forest management for over 200 years and

31 constitutes the basis for forest ecology and environmental policymaking today (Corona, Chirici,

32 McRoberts, Winter, \& Barbati, 2011; Newnham et al., 2015). With increasing recognition of

33 forest multifunctionality, the use of forest inventory data has expanded from quantifying timber

34 productivity to assessing forest biodiversity and carbon sequestration (Corona et al., 2011).

35 Spatial data of trees in forest inventory plots allow detailed analyses of forest dynamics (Tatsumi,

36 Cadotte, \& Mori, 2019; Tatsumi, Iritani, \& Cadotte, 2021) and species coexistence (Kunstler et

37 al., 2015; Tatsumi, Owari, \& Mori, 2016). As such, forest inventories offer a fundamental step

38 toward studying forest ecology and understanding the benefits forests provide to humanity.

Terrestrial laser scanning (TLS) is becoming increasingly popular as an alternative means

40 to conventional forest inventory methods (Newnham et al., 2015; Liang et al., 2016). By gauging

41 the distances to multiple points on the surrounding object surfaces, TLS acquires 3D point clouds

42 from which trees' sizes and spatial distributions can be rapidly estimated. However, the high cost

43 of TLS devices, typically priced at over US $\$ 40,000$, has put them out of reach for many potential

44 users (Eitel, Vierling, \& Magney, 2013). The heaviness of TLS devices has also been a challenge,

45 making their transportations to/within some areas difficult as well as adding shipping costs (Eitel

46 et al., 2013). The need for specialized computer programs has further limited the TLS user base

47 (Dassot, Constant, \& Fournier, 2011; Bunting, Armston, Lucas, \& Clewley, 2013). Broader

48 acceptance of TLS as a valid alternative to conventional methods thus requires affordable and

49 efficient hardware and software (Newnham et al., 2015).

Since 2020, Apple Inc. (Cupertino, CA, USA) have introduced a light detection and ranging

51 (LiDAR) sensor in some iPhone and iPad models, namely iPhone 12 Pro, iPhone 12 Pro Max,

52 iPhone 13 Pro, iPhone 13 Pro Max, iPad Pro 2020, and iPad Pro 2021 (as of December 2021). 
53 Compared to other TLS devices in the market, the LiDAR-equipped iPhones/iPads are available

54 at cheaper prices (US $\geq \$ 749$ ) and are lighter in weight (187-684 g). These iPhones/iPads have

55 been found suitable for acquiring 3D point clouds in forests (Gollob, Ritter, Kraßnitzer, Tockner,

56 \& Nothdurft, 2021; Mokroš et al., 2021). However, to derive tree information (e.g., stem

57 diameter) from the acquired point clouds, one must conduct post-analyses on a separate device

58 using multiple software with command-line interfaces, due to the current lack of dedicated

59 iPhone/iPad application programs.

Here, we introduce ForestScanner, a free application that allows LiDAR-based forest

61 inventories by means of iPhones/iPads. As the user scans trees with the device by walking in the

62 field, ForestScanner estimates the stem diameters and spatial coordinates in real-time (Figure 1;

63 see also Video S1 at FigShare, https://doi.org/10.6084/m9.figshare.17161823.v1). The users can

64 visualize, check, and share the results of scanning in situ. These features of ForestScanner allow

65 rapid forest inventories and increase the accessibility to TLS for people beyond specialists.

\section{2 | FORESTSCANNER APP}

\section{$68 \quad 2.1 \mid$ Description}

69 The ForestScanner application is available for free on the App Store (https://www.apple.com/appstore). The application operates on the above-mentioned iPhone/iPad models that have a time-of-

71 flight LiDAR sensor. As the user moves the device, ForestScanner scans objects within a distance

72 of $5 \mathrm{~m}$ (i.e., the maximum scanning range of the sensor), acquiring a 3D point cloud of the

73 surrounding object surfaces. When using ForestScanner, the point cloud and 3D triangle meshes

74 appear on the screen in real-time, allowing users to visually recognize the scanned surfaces

75 (Figure 1a; Video S1). The point cloud is colorized with RGB information collected by the 
77 coordinates from the starting point based on the inertial measurement unit (IMU) (i.e., dead

78 reckoning). The absolute location (i.e., geographic coordinates) of the starting point is determined

79 by the GNSS built into the iPhone/iPad.

80 Similar to other existing methods for TLS-based forest measurements (e.g., Dassot et al.,

81 2011; Pueschel et al., 2013; Calders et al., 2015), ForestScanner performs stem detections and

82 circle fitting as separate tasks. First, real-time object detection of individual stems is conducted.

83 Circles are then fitted to the cross-sections of the segmented trees in the point cloud space by

84 means of the Levenberg-Marquardt non-linear least-squares method (Björck, 1996). The

85 recommended lower threshold of target tree diameter is 5-10 cm (Gollob et al., 2021; Mokroš et

86 al., 2021), as the current iPhone/iPad LiDAR sensor has limited capability of detecting objects

87 that are a few centimeters in size (Vogt, Rips, \& Emmelmann, 2021). Diameters at breast height

88 (DBH) (or any other height of one's choice) and coordinates of the trees are estimated as the

89 diameters and centers of the fitted circles.

90

$91 \quad 2.2 \mid$ Usage

92 - Tap the "Record" button on the screen to start scanning. A crosshair symbol, a slider bar, and

93 the "Tree", "Pause", "Back", "Note", "Stop", “Tree species", and "Album" buttons will

94 appear on the screen (Figure 1a, Video S1).

95 - While walking in the field, scan target trees individually from a distance of $0.3 \mathrm{~m}$ to $5 \mathrm{~m}$. Use

96 the slider bar to fix the maximum scanning distance.

97 - For each tree, overlay the crosshair symbol at the height where the diameter should be

98 measured (e.g., $1.3 \mathrm{~m}$ ). Then tap the "Tree" button to identify the stem. The stem diameter

99 will appear on the screen in an augmented-reality manner (Figure 1a).

100 - If multiple trees are to be scanned in one survey, move to the next tree while scanning. 
101 Alternatively, tap the "Pause" button, move to the next tree, and tap the "Record" button to

102 resume. During the pause, the LiDAR sensor stops scanning while the IMU continues to

103 keep track of the device's location. Using the pause function helps to reduce the file size of

104 the point cloud.

105 - Tap the "Tree species" button to enter the tree species (optional). The tree species can be

106 entered by using either the screen keyboard or voice input (i.e., iPhone/iPad's microphone).

107 - Tap the "Back" button to delete the tree previously scanned.

108 - Tap the "Note" button to add a note about the tree previously scanned.

109 - Tap the "Stop" button to end the survey. The acquired point cloud will be displayed on the

110 screen (Figure 1b).

111 - After the survey, tap the "Tree List" button to show the list of tree ID, diameters, geographic
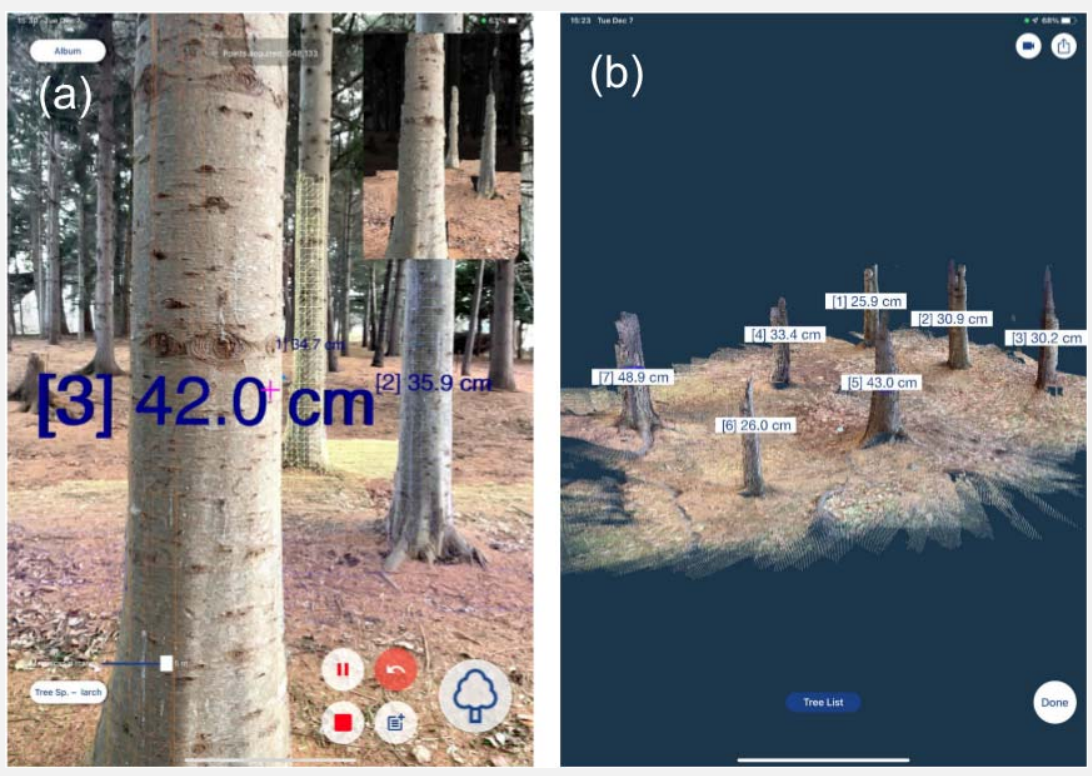

Figure 1. ForestScanner screenshots. (a) As the trees are scanned, stem diameters appear instantly on the screen in an augmented-reality manner. (b) An example of point cloud and tree diameters obtained by ForestScanner. See also Video S1 (https://doi.org/10.6084/m9.figshare.17161823.v1) for a demonstration of the app's usage. 
112 coordinates, tree species, and notes. The list can be sent to other devices by email or AirDrop.

113 - Tap the "Album" button to show the results of previous surveys.

114

\section{3 | FIELD APPLICATIONS}

\section{$116 \quad 3.1 \mid$ Data collection and analyses}

117 We applied ForestScanner to a 1 ha $(100 \mathrm{~m} \times 100 \mathrm{~m})$ plot composed of twenty-five 0.04 ha $(20 \mathrm{~m}$

$118 \times 20 \mathrm{~m})$ subplots in the Hokkaido Research Center, Forestry and Forest Products Research

119 Institute, Japan (4259'57" N, 141 23'29" E) (Figure 2a). The plot encompasses mosaics of

(a)

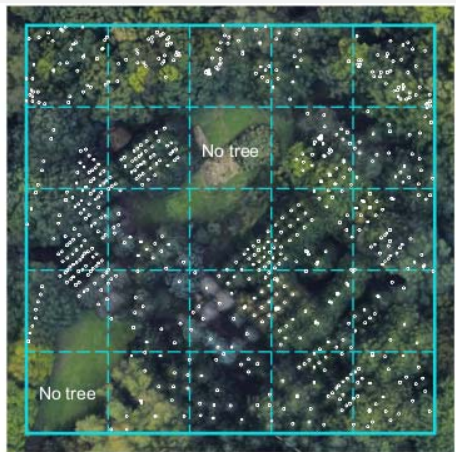

(c)

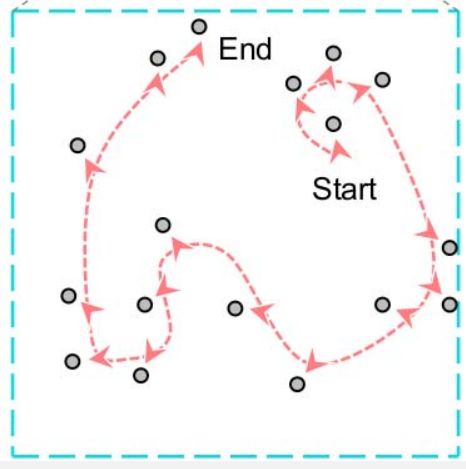

(b)

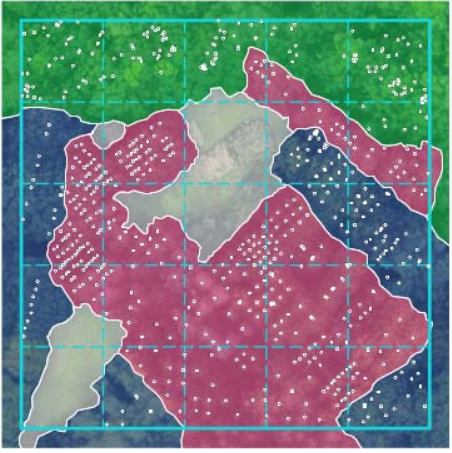

$100 \mathrm{~m} \times 100 \mathrm{~m}$ plot

$20 \mathrm{~m} \times 20 \mathrm{~m}$ subplots

Natural forests

Conifer plantations

Broadleaf plantations

Canopy gaps

Trees

Scanning path

Scanning directions

Figure 2. Overview of the study plot. (a, b) The $100 \mathrm{~m} \times 100 \mathrm{~m}$ plot consisting of 20 $\mathrm{m} \times 20 \mathrm{~m}$ subplots and multiple forest types. (c) An example of the path walked during scanning and the directions from which trees were scanned by ForestScanner. Background image: Google Earth. 
120 multiple forest types including conifer and broadleaf plantations of different stand ages and

121 natural secondary forests (Figure 2b).

122 All the trees with $\mathrm{DBH} \geq 5 \mathrm{~cm}$ were measured and mapped using an iPhone 13 Pro and an

123 iPad Pro 2021. We defined the 0.04 ha subplots as the unit of scanning, with consideration given

124 to the plot sizes often adopted in national forest inventory programs (Paul, Kimberley, \& Beets,

125 2019) and previous iPhone/iPad assessments which used 0.016 ha (Gollob et al., 2021) and 0.063

126 ha plots (Mokroš et al., 2021). Using each device, the surveyor (author ST) scanned the trees

127 consecutively in each subplot (Figure 2c). Scanning was conducted in 23 subplots since there

128 were no trees in two subplots (Figure 2a).

129 To compare the results of ForestScanner with conventional methods, we measured the

130 DBH using a diameter tape. The measurement was conducted by one measurer and one note-

131 taker (authors ST and NF). We also measured the spatial coordinates of the trees using a

132 combination of laser range-finder, electronic inclinometer, and electronic compass (Impulse 200

133 and MapStar Compass Module; Laser Technology Inc., Centennial, CO, USA) attached to a

134 personal digital assistant (PA692; Unitech Electronics Co., Ltd., New Taipei City, Taiwan).

135 Using this surveying system, two people conducted the survey; one person operating the system

136 and the other holding a reflection board by the trees (authors ST and NF). We conducted closed

137 traverses, starting from a control point with known geographic coordinates (i.e., a corner of the

138 plot, a midpoint of the plot edges, or the plot center) and either returning to the same point or

139 closing on another control point. Trees were mapped by measuring the distances and angles from

140 the traverse stations.

141 We assessed the congruence between the DBH measured by ForestScanner and a diameter

142 tape based on the coefficient of determination $\left(\mathrm{R}^{2}\right)$ and the root mean squared error (RMSE). The

143 results of tree mapping by ForestScanner and the conventional surveying system were compared 
144 using Mantel $\mathrm{R}^{2}$; i.e., we assessed the association between two matrices representing the spatial

145 distances among trees within each subplot. We assessed the trees' relative distances but not their

146 absolute locations because the latter depends not only on the accuracy of the IMU but also the

147 GNSS built into the iPhone/iPad (see 2.1 Description) and on various field settings (e.g.,

148 availability of known points for dead reckoning) which were beyond the scope of this study.

\section{$150 \quad 3.2 \mid$ Accuracy of scanning}

151 The DBH of all the 672 trees $(\geq 5 \mathrm{~cm})$ in the plot were successfully measured by ForestScanner.

152 Tree detection rate was $100 \%$ as the trees were scanned independently on-site. The DBH

153 measured by ForestScanner and a diameter tape were in good agreement; the $\mathrm{R}^{2}$ values were

1540.963 using iPhone and 0.961 using iPad (Figure 3a, 3b). The RMSE were $2.27 \mathrm{~cm}$ using iPhone

155 and $2.32 \mathrm{~cm}$ using iPad. Our results were comparable with the results from previous studies

156 based on manual post-analyses of iPhone/iPad-acquired point clouds; detection rate $=\sim 80 \%, \mathrm{R}^{2}=$

157 0.973, and RMSE = 3.13-4.51 cm (Gollob et al., 2021; Mokroš et al., 2021). Spatial distances

158 among trees measured by ForestScanner and the conventional surveying system were almost

159 identical; the mean Mantel $\mathrm{R}^{2}$ (averaged across 23 subplots) were 0.999 using both iPhone and

160 iPad (Figure 3c, 3d).

\section{$162 \quad 3.3 \mid$ Time spent for scanning}

163 Using an iPhone, it took $1 \mathrm{~h} 39 \mathrm{~min}(=1.66 \mathrm{~h})$ for one person to measure the DBH and

164 coordinates of all the 672 trees in the plot (Table 1$)$. It took $1 \mathrm{~h} 38 \mathrm{~min}(=1.64 \mathrm{~h})$ with an iPad to

165 do the same things. Using a diameter tape, it took $3 \mathrm{~h} 13 \mathrm{~min}(=3.21 \mathrm{~h})$ for two people to

166 measure the DBH (Table 1). To map the trees using the conventional surveying system, it took 8

$167 \mathrm{~h} 53 \mathrm{~min}(=8.88 \mathrm{~h})$ for two people (Table 1$)$. As such, compared to the conventional tools, 
168 ForestScanner reduced the person-hours (i.e., the number of workers $\times$ hours) required for

169 measuring DBH to $25.7 \%(=1.65 / 6.42)$, for mapping trees to $9.3 \%(=1.65 / 17.76)$, and for doing

170 both to $6.8 \%(=1.65 / 24.18)$.

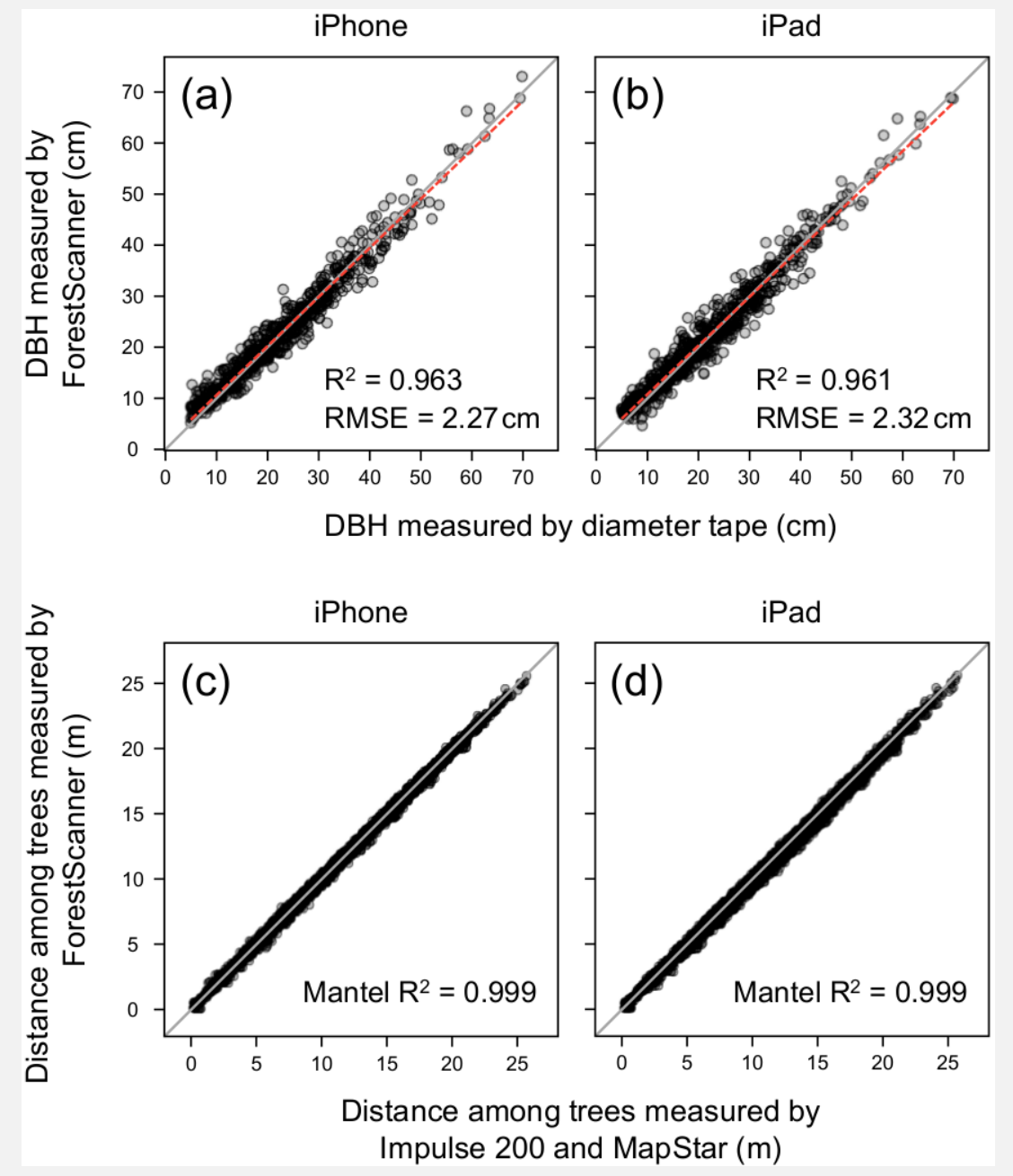

Figure 3. Measurement accuracy of ForestScanner. $(a, b)$ Congruence between DBH measured by a diameter tape and ForestScanner using iPhone and iPad. The red dashed lines indicate regression lines. (c, d) Congruence between the distances among trees measured by a conventional surveying system (a combination of Impulse 200 and MapStar Compass Module) and ForestScanner using iPhone and iPad. 


\section{4 | CONCLUSIONS}

173 ForestScanner is a free iPhone/iPad application that allows on-site, real-time measurements of

174 tree diameters and spatial coordinates. We demonstrated that the application requires less labor

175 and time in the field compared to conventional forest inventory tools (Table 1). Tree diameters

176 and coordinates measured by ForestScanner and conventional tools showed good agreement

177 (Figure 3). Future works require further evaluations of the application's performance under

178 various environmental conditions and comparisons with other TLS devices. ForestScanner is

179 intuitively operable and does not require any manual post-analyses of 3D point clouds, providing

180 access to LiDAR technology for non-specialists (e.g., students). We believe that ForestScanner

181 will facilitate TLS-based resource assessments and biodiversity monitoring in forests worldwide.

\section{AUTHORS' CONTRIBUTIONS}

184 ST conceived the study. KY developed the application with inputs from ST and NF. ST and NF

Table 1. The number of workers and time spent to measure the DBH and spatial coordinates of 672 trees in a 1 ha plot using ForestScanner (iPhone and iPad), a diameter tape, and a conventional surveying system (a combination of Impulse 200 and MapStar Compass Module).

\begin{tabular}{lllll}
\hline & iPhone & iPad & $\begin{array}{l}\text { Diameter } \\
\text { tape }\end{array}$ & $\begin{array}{l}\text { Impulse 200 and } \\
\text { MapStar }\end{array}$ \\
\hline Items measured & $\begin{array}{l}\text { DBH and } \\
\text { coordinates }\end{array}$ & $\begin{array}{l}\text { DBH and } \\
\text { coordinates }\end{array}$ & DBH & Coordinates \\
Number of workers & 1 & 1 & $2^{*}$ & $2^{\dagger}$ \\
Time spent (hours) & 1.66 & 1.64 & 3.21 & 8.88 \\
Workers $\times$ hours & 1.66 & 1.64 & 6.42 & 17.76 \\
\hline
\end{tabular}

*A measurer and a note-taker.

${ }^{\dagger}$ An operator and a reflection-board holder. 
185 conducted the fieldwork. ST analyzed the data and wrote the manuscript. All authors contributed

186 to manuscript revisions.

188 DATA ACCESSIBILITY

189 Tree diameter and coordinate data obtained by iPhone, iPad, and conventional methods will be

190 made available at FigShare upon acceptance. A demonstration of ForestScanner usage (Video S1)

191 is available at https://doi.org/10.6084/m9.figshare.17161823.v1.

193 ACKNOWLEDGEMENTS

194 This work was supported by the Japan Society for the Promotion of Science (Grant-in-Aid for

195 Early-Career Scientists 21K14880) and the Council for the Development of Smart Forestry EZO

196 Model.

\section{REFERENCES}

199 Björck, Å. (1996). Numerical methods for least squares problems. Society for Industrial and 200 Applied Mathematics.

201 Bunting, P., Armston, J., Lucas, R. M., \& Clewley, D. (2013). Sorted pulse data (SPD) library.

204 Calders, K., Newnham, G., Burt, A., Murphy, S., Raumonen, P., Herold, M., ... Kaasalainen, M.

205 (2015). Nondestructive estimates of above-ground biomass using terrestrial laser scanning. 206 Methods in Ecology and Evolution, 6, 198-208.

207 Corona, P., Chirici, G., McRoberts, R. E., Winter, S., \& Barbati, A. (2011). Contribution of 208 large-scale forest inventories to biodiversity assessment and monitoring. Forest Ecology and 
Management, 262, 2061-2069.

210 Dassot, M., Constant, T., \& Fournier, M. (2011). The use of terrestrial LiDAR technology in

211 forest science: Application fields, benefits and challenges. Annals of Forest Science, 68,

$212959-974$.

213 Eitel, J. U. H., Vierling, L. A., \& Magney, T. S. (2013). A lightweight, low cost autonomously

214 operating terrestrial laser scanner for quantifying and monitoring ecosystem structural

215 dynamics. Agricultural and Forest Meteorology, 180, 86-96.

216 Gollob, C., Ritter, T., Kraßnitzer, R., Tockner, A., \& Nothdurft, A. (2021). Measurement of

217 forest inventory parameters with Apple iPad Pro and integrated LiDAR technology. Remote

$218 \quad$ Sensing, 13, 3129.

219 Kunstler, G., Falster, D., Coomes, D. A., Hui, F., Kooyman, Robert, M., Laughlin, D. C., ...

220 Ruiz-Benito, P. (2015). Plant functional traits have globally consistent effects on

221 competition. Nature, 529, 1-15.

222 Liang, X., Kankare, V., Hyyppä, J., Wang, Y., Kukko, A., Haggrén, H., ... Vastaranta, M. (2016). Terrestrial laser scanning in forest inventories. ISPRS Journal of Photogrammetry and Remote Sensing, 115, 63-77.

Mokroš, M., Mikita, T., Singh, A., Tomaštík, J., Chudá, J., Wężyk, P., ... Liang, X. (2021). Novel low-cost mobile mapping systems for forest inventories as terrestrial laser scanning alternatives. International Journal of Applied Earth Observation and Geoinformation, 104, 102512.

Newnham, G. J., Armston, J. D., Calders, K., Disney, M. I., Lovell, J. L., Schaaf, C. B., ... Mark Danson, F. (2015). Terrestrial laser scanning for plot-scale forest measurement. Current Forestry Reports, 1, 239-251.

232 Paul, T. S. H., Kimberley, M. O., \& Beets, P. N. (2019). Thinking outside the square: Evidence 

in Ecology and Evolution, 10, 381-388.

235 Pueschel, P., Newnham, G., Rock, G., Udelhoven, T., Werner, W., \& Hill, J. (2013). The

236 influence of scan mode and circle fitting on tree stem detection, stem diameter and volume extraction from terrestrial laser scans. ISPRS Journal of Photogrammetry and Remote

$238 \quad$ Sensing, 77, 44-56.

239 Tatsumi, S., Cadotte, M. W., \& Mori, A. S. (2019). Individual-based models of community

240 assembly: Neighbourhood competition drives phylogenetic community structure. Journal of

$241 \quad$ Ecology, 107, 735-746.

242 Tatsumi, S., Iritani, R., \& Cadotte, M. W. (2021). Temporal changes in spatial variation:

243 Partitioning the extinction and colonisation components of beta diversity. Ecology Letters,

$244 \quad 24,1063-1072$.

245 Tatsumi, S., Owari, T., \& Mori, A. S. (2016). Estimating competition coefficients in tree

246 communities: A hierarchical Bayesian approach to neighborhood analysis. Ecosphere, 7, $247 \quad \mathrm{e} 01273$.

248 Vogt, M., Rips, A., \& Emmelmann, C. (2021). Comparison of iPad Pro®’s LiDAR and 